\title{
Novel HSPG 2 mutations causing Schwartz-Jampel syndrome type 1 in a Chinese family: A case report
}

\author{
WENJIN YAN $^{1 *}$, JIN DAI $^{1 *}$, DONGQUAN SHI ${ }^{1}$, XINGQUAN XU $^{1}$, XIAO HAN $^{1}$, \\ ZHIHONG XU ${ }^{1}$, DONGYANG CHEN ${ }^{1}$, HUAJIANG TENG ${ }^{2}$ and QING JIANG ${ }^{1}$ \\ ${ }^{1}$ Department of Sports Medicine and Adult Reconstructive Surgery, Drum Tower Hospital, \\ School of Medicine, Nanjing University, Nanjing, Jiangsu 210008; ${ }^{2}$ Laboratory for Bone and Joint Disease, \\ Model Animal Research Center (MARC), Nanjing University, Nanjing, Jiangsu 210093, P.R. China
}

Received November 2, 2017; Accepted March 5, 2018

DOI: $10.3892 / \mathrm{mmr} .2018 .9143$

\begin{abstract}
Schwartz-Jampel syndrome type 1 (SJS1) is a rare autosomal recessive disease caused by mutations in the gene heparan sulfate proteoglycan 2 (HSPG2; also known as basement membrane-specific heparin sulfate). In the present study, a 10-year-old female SJS1 proband from a Chinese family, who was diagnosed by X-ray and physical examination, was recruited. The key clinical features of the patient with SJS1 included short stature, joint contractures, pigeon breast, and myotonia that led to progressive stiffness of the face and limbs; barely discernible kyphosis was also noted. Genetic testing using whole exome sequencing and Sanger sequencing was performed for the proband and family members. A total of 2 novel mutations (c.8788G $>$ A; p.Glu2930Lys and c.11671+5G $>A$ ) in the HSPG2 gene were identified in the proband. The family members harboring 1 heterozygous mutation in $H S P G 2$ did not exhibit any skeletal abnormalities. The results of the present study suggested that the compound heterozygous mutations in HSPG2 may be responsible the induction of SJS1, and demonstrated the genotype-phenotype associations between mutations in the HSPG2 gene and clinical characteristics of SJS1.
\end{abstract}

\section{Introduction}

Schwartz-Jampel syndrome type 1 (SJS1; Online Mendelian Inheritance in Man \#255800) is a type of autosomal recessive skeletal dysplasia characterized by permanent myotonic

Correspondence to: Professor Qing Jiang, Department of Sports Medicine and Adult Reconstructive Surgery, Drum Tower Hospital, School of Medicine, Nanjing University, 321 Zhongshan Road, Nanjing, Jiangsu 210008, P.R. China

E-mail: qingj@nju.edu.cn

${ }^{*}$ Contributed equally

Key words: Schwartz-Jampel syndrome type 1, heparan sulfate proteoglycan 2, compound heterozygous mutations, whole exome sequencing myopathy and skeletal dysplasia, which result in short stature, dystrophy of epiphyseal cartilages, joint contractures, blepharophimosis, unusual pinnae, myopia and pigeon breast. In addition, myotonic (leading to progressive stiffness of the face and limbs), persistent bowing of the limbs, and severe kyphoscoliosis may develop. Electromyograms exhibit continuous discharges at rest (1-3). Furthermore, walking becomes increasingly more difficult and secondary contractures of the large joints may develop in patients with SJS1; by late adolescence, severely affected patients may be confined to a bed and a wheelchair (2). Adult height varies from 140 to $170 \mathrm{~cm}$, and $25 \%$ of patients with SJS1 present with mental retardation $(4,5)$. In addition, procainamide therapy has been shown to help muscle function (6).

SJS1 is caused by mutations in heparan sulfate proteoglycan 2 (HSPG2; also known as basement membrane-specific heparin sulfate). The HSPG2 gene encodes the perlecan protein, a ubiquitous HSP, which serves essential roles in multiple biological activities, including forming the vascular extracellular matrix, maintaining endothelial barrier function, promoting growth factor and being a potent inhibitor of smooth muscle cell proliferation $(7,8)$. Alternative splicing of this gene results in multiple transcript variants, which may cause SJS1, Silverman-Handmaker type dyssegmental dysplasia, and tardive dyskinesia (9).

In the present study, one SJS1 proband from a Chinese family, who was diagnosed by X-ray and physical examination, was recruited and genetic sequencing was performed. Our study suggested that the compound heterozygous mutations in HSPG2 were responsible for SJS1 and demonstrated the genotype-phenotype relationship between mutations in the HSPG2 gene and characteristics of SJS1.

\section{Case report}

The Ethics Committee of Nanjing Drum Tower Hospital (Jiangsu, China) approved the present study. Written informed consent was obtained from all subjects or their parents/guardians. The 10 -year-old female proband was recruited to the present study for genetic testing between December 2016 and June 2017. The proband was the only child born to healthy parents of Chinese Han descent; there was no 
SJS1 or other skeletal diseases in the proband's family history. Upon physical examination of the proband, a peculiar facial appearance with pursed lips was noted. A small mouth, blepharophimosis, unusual pinnae and myopia were not presented (Fig. 1A); however, severe kyphoscoliosis, and progressive stiffness of the face, limbs and hands were observed in the proband (Fig. 1B-D). In addition, the patient did not exhibit anterior hypoplasia of cervical bodies with cervical kyphosis. At the age of 2, the proband exhibited hypotonia with poor muscle bulk and proximal leg weakness. Myotonic features, which resulted in progressive stiffness, were noticed during the proband's early childhood. At the age of 8 , the proband began to suffer from contractures of the large joints, including the elbow, knee and hip, and walking became difficult; a lordotic gait was also present at this time. The results of neurological and other examinations were within the normal range, and the proband's height was $85,3.5 \mathrm{~cm}$ below than the average height of Han Chinese girls of the same age.

$\mathrm{X}$-ray imaging performed when the patient was 10 years of age revealed micrognathia and a narrow upper thoracic inlet (Fig. 2). No flattened vertebral bodies with regular end-plates were noted (Fig. 2). There was significant kyphoscoliosis, progression of epiphyseal changes and generalized osteopenia. Bowed femora and large round capital femoral epiphyses were also observed (Fig. 2).

These radiographic results of the patient were similar to those described in previous reports of SJS1 $(1,10,11)$; however, due to the clinical and radiographic similarities of skeletal abnormalities, patients with other skeletal diseases, such as Stuve-Wiedemann syndrome or focal femoral hypoplasia-unusual facies syndrome, may be misdiagnosed with SJS1. Therefore, the present study performed molecular analysis of the patient and parents.

Once written informed consent was obtained, genomic DNA was extracted from the peripheral blood of the patient and family members using a Qiagen DNA Mini kit (Qiagen GmbH, Hilden, Germany). Whole exome sequencing (WES) of the proband was conducted. Briefly, genomic DNA was divided into smaller fragments of 200-250 bp using an ultrasonic instrument (Covaris LE220; Covaris, Inc., Woburn, MA, USA). Subsequently, purification with Ampure Beads (Beckman Coulter, Inc., Brea, CA, USA) was performed to add poly A/joint reaction to the end of the purified DNA fragments. The gene-trapping chip (Roche NimbleGen, Madison, WI, USA) was hybridized with the purified DNA fragments of the proband. Following hybridization, captured DNA was sequenced with an Illumina HiSeq2500 Analyzer (Illumina, Inc., San Diego, CA, USA) and read using Illumina Pipeline software (version 1.3.4; Illumina, Inc.). The present study used BWA v0.59 (12) to align sequence reads to the human genome reference (build 37) and removed duplicated reads from subsequent analyses. Sequence variants were identified via comparisons with the NCBI reference sequence NM 005529.5 and annotated by the current version of ANNOVAR (2017 Jul 16; annovar.openbioinformatics.org/en/latest/). The 20X coverage for the RefSeq coding region was $98.31 \%$ (Fig. 3).

A novel missense mutation (c.8788 G>A; p.Glu2930Lys) in exon 67 of $H S P G 2$ and a mutation in intron 85 (c.11671+5G $>\mathrm{A})$ the splicing region, were detected in the proband (Fig. 4A). One guanine ribonucleotide was altered to an adenine ribonucleotide in codon 8788 , which caused a change in the reading frame from glutamine to lysine. The Polyphen-2 tool (13) predicted the mutation as likely to cause damage to the HSPG2 protein function (Table I). To the best of our knowledge, the mutations c. $8788 \mathrm{G}>\mathrm{A}$ and c. $11671+5 \mathrm{G}>\mathrm{A}$ have not been reported in SJS1 previously. The mutation c.8788G $>$ A; p.Glu2930Lys was located in the domain Immunoglobulin_2 in HSPG2, while the mutation $c .11671+5 \mathrm{G}>\mathrm{A}$ was present in the splicing region near the Epidermal Growth Factor Calcium-binding domain. These 2 mutations may result in the loss-of-function of HSPG2. In the 1,000 Genomes database (www.internationalgenome.org), dbSNP database (www.ncbi.nlm.nih.gov/SNP/), Exome Sequencing Project 6500 database (evs.gs.washington. edu/EVS/) and Exome Aggregation Consortium EAS database (exac.broadinstitute.org), the allele frequencies of the c. $8788 \mathrm{G}>\mathrm{A}$ mutation were $0,0,0$ and 0.000155 , respectively, while the allele frequencies of $c .11671+5 \mathrm{~g}>\mathrm{A}$ mutation were 0, 0, 0.000077 and 0.01684, respectively (Table I).

In the present study, Sanger sequencing was also performed for the proband's family, which confirmed the compound heterozygous mutation in the proband and the heterozygous status of the father and mother (Fig. 4A); there was no evidence of SJS1 or of other skeletal diseases in the proband's parents. Furthermore, the mutations c.8788G $>$ A; p.Glu2930Lys and c.11671+5G $>$ A were highly conserved among a diverse range of species (Fig. 4B). Thus, the present study identified 2 novel HSPG2 mutations in a child case of SJS1.

\section{Discussion}

SJS1 is a rare, autosomal recessive progressive disorder that is characterized by clinical features including short stature, myotonic myopathy, dystrophy of epiphyseal cartilages, joint contractures, blepharophimosis, unusual pinnae, myopia, pigeon breast, and progressive stiffness of the face and limbs (14-16). This disorder can be further divided into the subtypes: SJS1A, a milder phenotype with an onset during infancy to early childhood and relatively milder chondrodysplasia; and SJS1B, a more severe phenotype with neonatal onset and significant chondrodysplasia (17). Mask-like facies typically manifest as blepharophimosis, with a pursed mouth and a fixed face with a sad appearance. Chondrodysplasia consists of metaphyseal widening, slender diaphysis and kyphoscoliosis (18).

Recessive inheritance is consistent with the loss-of-function nature of the 2 mutations (c.8788 G>A; p.Glu2930Lys, and c. $11671+5 \mathrm{G}>\mathrm{A}$ ) and the previously reported HSPG 2 mutations in SJS (14-18). In total, $<40$ patients with SJS have been reported and no genotype-phenotype correlation is apparent. Although the splicing variation c. $11671+5 \mathrm{G}>\mathrm{A}$ is a non-coding variant, it is highly conserved among diverse species and several splicing-site variants in HSPG2 have been reported to cause the aberrant splicing in the exon region $(14,15,18)$, which form a considerable proportion of the reported variants. In addition, the splice variation c. $11671+5 \mathrm{G}>\mathrm{A}$ in intron 85 of the HSPG2 gene could highly affect the 5' splice site, the base 5 nucleotides downstream of intron 85 , increasing the likelihood of reducing the content of effective mRNA that codes for the correct protein. Finally, by combining the specific clinical information and genetic evidence, the present study confirmed the diagnosis of SJS1 in the proband. 

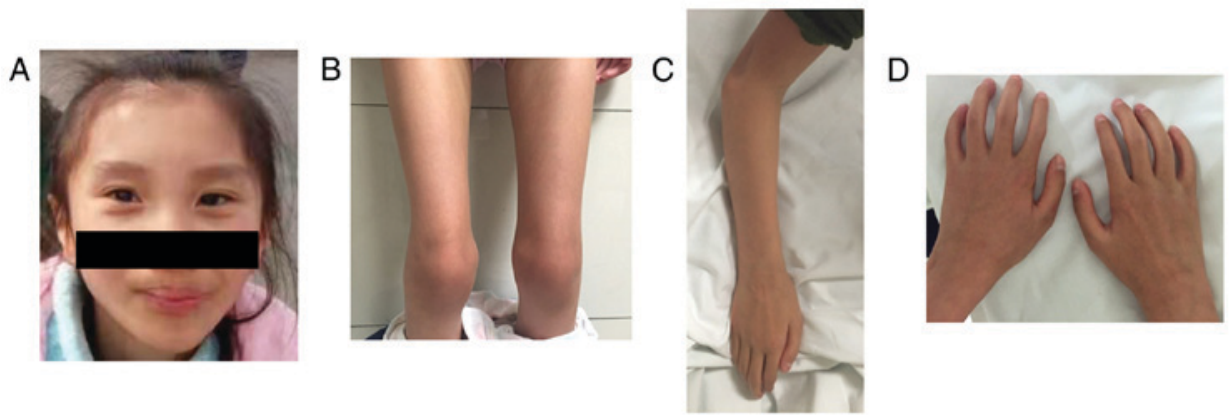

Figure 1. Clinical features of the patient with Schwartz-Jampel syndrome type 1. (A) Peculiar facial appearance with pursed lips and blepharophimosis. (B) Bowed legs. (C) Hand of patient at age 10. The interphalangeal joints exhibit contracture. (D) Progressive stiffness and joint contractures were presented.
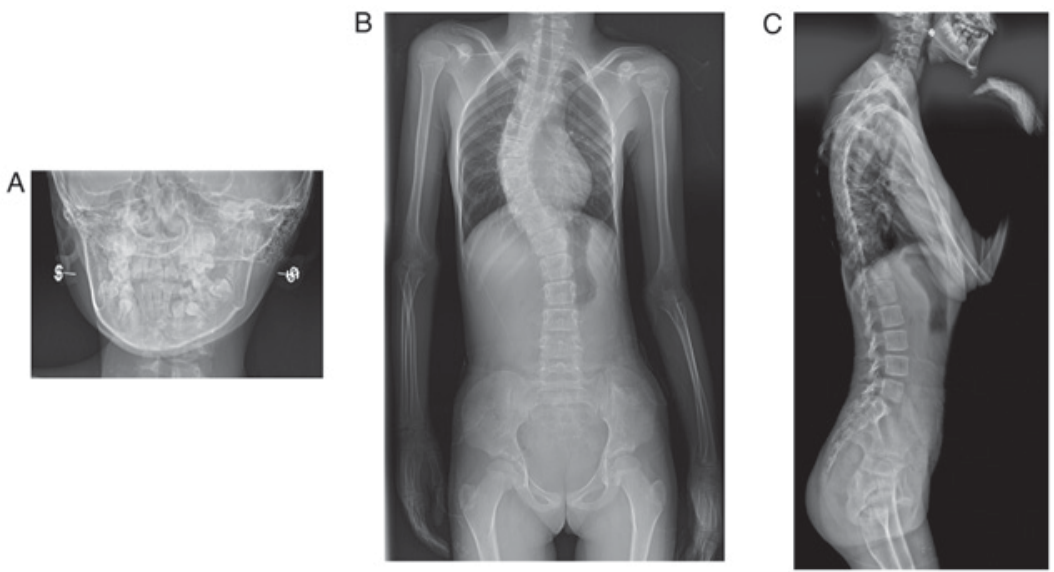

Figure 2. Radiographic features of the 10-year-old female patient (the proband) with Schwartz-Jampel syndrome type 1. (A) Micrognathia, and the (B and C) Pectus and Pelvis. A narrow upper thoracic inlet was noted, as well as severe kyphoscoliosis, bowing of the long bones, epiphyseal, metaphyseal and hip dysplasia. In addition, a bowed femora and large round capital femoral epiphyses were observed.

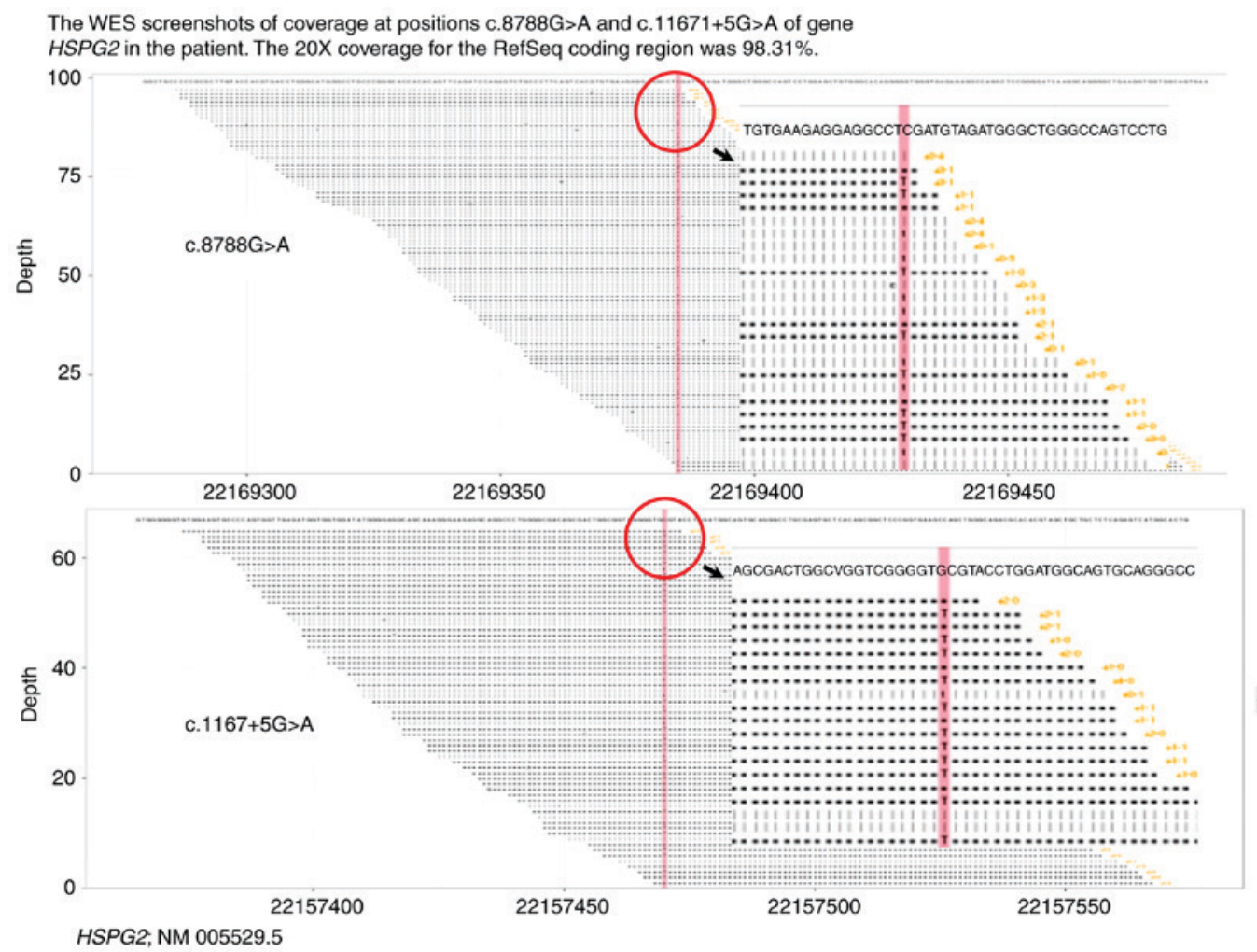

Figure 3. The WES screenshots of coverage at positions c.8788G $>$ A and c.11671+5G $>$ A of gene HSPG2 in the patient. The 20X coverage for the RefSeq coding region was $98.31 \%$. 
Table I. Identification of single nucleotide variations in the heparan sulfate proteoglycan 2 gene through whole exome sequencing.

\begin{tabular}{|c|c|c|}
\hline Variable & c. $11671+5 \mathrm{G}>\mathrm{A}$ mutation & c.8788G $>$ A mutation \\
\hline Chromosome & 1 & 1 \\
\hline Position & $22,157,470$ & $22,169,385$ \\
\hline Predicted protein variants & - & p.Glu2930Lys \\
\hline Variants type & Het & Het \\
\hline Gene region & Splicing & Exon \\
\hline Ref Seq & NM_005529.5 & NM_005529.5 \\
\hline SNP ID & rs77527456 & rs368020528 \\
\hline 1000g_chbs & - & - \\
\hline dbSNP & 0 & - \\
\hline ExAC_EAS & 0.01684 & 0 \\
\hline ESP6500 & 0.000077 & 0.000155 \\
\hline Polyphen & - & 'Probably damaging' \\
\hline
\end{tabular}

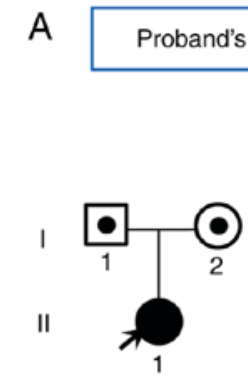

B

Homo sapiens
Macaca fascicularis
Pongo abelii
Gorilla gorilla gorilla
Nomascus leucogenys
Pan troglodytes
Rhinopithecus roxellana
Mustela putorius
Fukomys damarensis
Ceratotherium simum simum

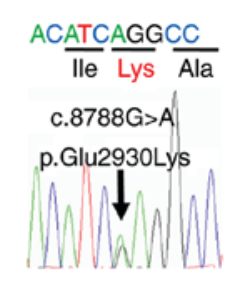

GGTACGCACCC

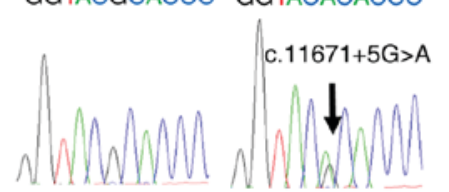

I-1

$\mathrm{I}-2$

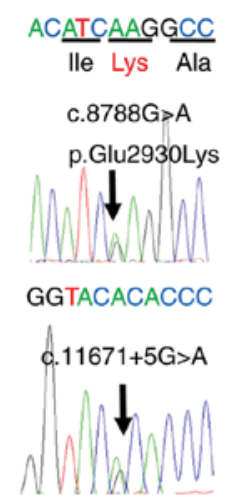

II-1

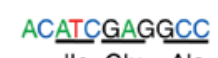

Ile Glu Ala

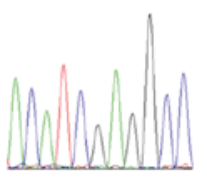

GGTACGCACCC

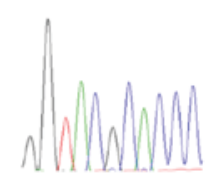

Control

Glu2930

APGLAQPIYIEASSSHVTEG

APGLAQPIYIEASSSHVTEG

APGLAQPIYIEASSSHVTEG

APGLAQPIYIEASSSHVTEG

APGLAQPIYIEASSSHVTEG

APGLAQPIYIEASSSHVTEG

APGLAQPIYIEASSSHVTEG

APGLAQPIYIEASSSHVAE

APGLAQPIYIEASSSHVAEG

APGLAQPIYIEASSSHLTEG

HSPG2; NM 005529.5

Figure 4. Pedigree of a family with Schwartz-Jampel syndrome type 1 and amino acid sequence alignment of $H S P G 2$. (A) Pedigree of the family with autosomal recessive transmission, and DNA sequencing of the $H S P G 2$ gene in the family for the mutations c. $8788 \mathrm{G}>\mathrm{A}$ and c. $11671+5 \mathrm{G}>\mathrm{A}$. The black circle and arrow indicate the proband. The proband (II-1) was a compound heterozygote for the mutation. The parents were carriers; indicated by white shapes with black circles in the center. Circles indicate females and squares represent males. (B) The amino acid alignment around the missense mutation (c.8788G $>\mathrm{A}$; p.Glu2930Lys) identified in the family was evolutionally conserved across a variety of different species. HSPG2, heparan sulfate proteoglycan 2.

The present study utilized WES, which allows molecular results to be obtained faster, particularly when traditional specific diagnosis takes a longer time to sequence a large gene. $H S P G 2$ is a very large gene, consisting of 97 exons, and encodes for the protein Perlecan, which is known to serve an important role in maintaining cartilaginous tissue integrity and regulating muscle excitability (2). Exome sequencing provides the ability to identify rare variants, analyze the candidate gene and to check for the presence of mutations in the genes at the same time $(19,20)$. In the present study, the HSPG2 mutation 
spectrum was further expanded, thus contributing to the earlier detection of the disease, which may provide significant benefit to the patient and their family. In addition, it may increase awareness of the growing number of SJS1 patients for future clinical diagnosis.

\section{Acknowledgements}

We thank the patient and her families who donated their blood samples for this study.

\section{Funding}

The present study was supported by The Projects of International Cooperation and Exchanges NSFC (grant no. 81420108021), Major Projects of NSFC (grant no. 8173000209), Excellent Young Scholars NSFC (grant no. 81622033), Jiangsu Provincial Key Medical Center Foundation, Jiangsu Provincial Medical Talent Foundation and Jiangsu Provincial Medical Outstanding Talent Foundation.

\section{Availability of data and materials}

The datasets used and/or analyzed during the current study are available from the corresponding author on reasonable request.

\section{Authors' contributions}

JD, XX, DS, DC, ZX and QJ performed the physical examination and diagnosis of the patients. WY, XH and HT analyzed and interpreted the WES data, and were major contributors to writing and revising the manuscript. All authors read and approved the final manuscript.

\section{Ethics approval and consent to participate}

The Ethics Committee of Nanjing Drum Tower Hospital (Jiangsu, China) approved the present study. Written informed consent was obtained from all subjects or their parents/guardians.

\section{Consent for publication}

Written informed consent was obtained from the proband and their parents for the publication of the data and images presented in the present study.

\section{Competing interests}

The authors declare that they have no competing interests.

\section{References}

1. Mathur N and Ghosh PS: Schwartz-jampel syndrome. Pediatr Neurol 68: 77-78, 2017.

2. Nicole S, Davoine CS, Topaloglu H, Cattolico L, Barral D, Beighton P, Hamida CB, Hammouda H, Cruaud C, White PS, et al: Perlecan, the major proteoglycan of basement membranes, is altered in patients with Schwartz-Jampel syndrome (chondrodystrophic myotonia). Nat Genet 26: 480-483, 2000.
3. Jablecki $C$ and Schultz P: Single muscle fiber recordings in the Schwartz-Jampel syndrome. Muscle Nerve 5: S64-S69, 1982.

4. Arikawa-Hirasawa E, Le AH, Nishino I, Nonaka I, Ho NC, Francomano CA, Govindraj P, Hassell JR, Devaney JM, Spranger J, et al: Structural and functional mutations of the perlecan gene cause Schwartz-Jampel syndrome, with myotonic myopathy and chondrodysplasia. Am J Hum Genet 70: 1368-1375, 2002.

5. Rodgers KD, Sasaki T, Aszodi A and Jacenko O: Reduced perlecan in mice results in chondrodysplasia resembling Schwartz-Jampel syndrome. Hum Mol Genet 16: 515-528, 2007.

6. Kashkouli MB, Shahrzad S, Jazayeri AA and Abtahi MB: Treatment of blepharospasm in schwartz-jampel syndrome: Botulinum toxin A injection or surgery. Ophthal Plast Reconstr Surg 33 (3S Suppl 1): S47-S49, 2017.

7. Stum M, Girard E, Bangratz M, Bernard V, Herbin M, Vignaud A, Ferry A, Davoine CS, Echaniz-Laguna A, René F, et al: Evidence of a dosage effect and a physiological endplate acetylcholinesterase deficiency in the first mouse models mimicking Schwartz-Jampel syndrome neuromyotonia. Hum Mol Genet 17: 3166-3179, 2008

8. Kallunki P, Eddy RL, Byers MG, Kestilä M, Shows TB and Tryggvason K: Cloning of human heparan sulfate proteoglycan core protein, assignment of the gene (HSPG2) to 1p36.1----p35 and identification of a BamHI restriction fragment length polymorphism. Genomics 11: 389-396, 1991.

9. Dodge GR, Kovalszky I, Chu ML, Hassell JR, McBride OW, Yi HF and Iozzo RV: Heparan sulfate proteoglycan of human colon: Partial molecular cloning, cellular expression, and mapping of the gene (HSPG2) to the short arm of human chromosome 1. Genomics 10: 673-680, 1991.

10. Stum M, Davoine CS, Vicart S, Guillot-Noël L, Topaloglu H, Carod-Artal FJ, Kayserili H, Hentati F, Merlini L, Urtizberea JA, et al: Spectrum of HSPG2 (Perlecan) mutations in patients with Schwartz-Jampel syndrome. Hum Mutat 27: 1082-1091, 2006

11. Spranger J, Hall BD, Häne B, Srivastava A and Stevenson RE: Spectrum of Schwartz-Jampel syndrome includes micromelic chondrodysplasia, kyphomelic dysplasia, and Burton disease. Am J Med Genet 94: 287-295, 2000.

12. Li H and Durbin R: Fast and accurate short read alignment with Burrows-Wheeler transform. Bioinformatics 25: 1754-1760, 2009.

13. Kelley LA and Sternberg MJ: Protein structure prediction on the Web: A case study using the Phyre server. Nat Protoc 4: 363-371, 2009.

14. Iwata S, Ito M, Nakata T, Noguchi Y, Okuno T, Ohkawara B, Masuda A, Goto T, Adachi M, Osaka H, et al: A missense mutation in domain III in HSPG2 in Schwartz-Jampel syndrome compromises secretion of perlecan into the extracellular space. Neuromuscul Disord 25: 667-671, 2015.

15. Das Bhowmik A, Dalal A, Matta D, Kandadai RM, Kanikannan MA and Aggarwal S: Identification of a novel splice site HSPG2 mutation and prenatal diagnosis in Schwartz Jampel Syndrome type 1 using whole exome sequencing. Neuromuscul Disord 26: 809-814, 2016.

16. Shah M and Scavina M: Novel mutations in Schwartz-Jampel syndrome with successes in medical management. Neurology 84 (Suppl 14): P2.237, 2015.

17. Giedion A, Boltshauser E, Briner J, Eich G, Exner G, Fendel H, Kaufmann L, Steinmann B, Spranger J and Superti-Furga A: Heterogeneity in Schwartz-Jampel chondrodystrophic myotonia. Eur J Pediatr 156: 214-223, 1997.

18. Arikawa-Hirasawa E, Le AH, Nishino I, Nonaka I, Ho NC, Francomano CA, Govindraj P, Hassell JR, Devaney JM, Spranger J, et al: Structural and functional mutations of the perlecan gene cause Schwartz-Jampel syndrome, with myotonic myopathy and chondrodysplasia. Am J Hum Genet 70: 1368-1375, 2002 .

19. Lim BC, Yoo SK, Lee S, Shin JY, Hwang H, Chae JH, Hwang YS, Seo JS, Kim JI and Kim KJ: Hoyeraal-Hreidarsson syndrome with a DKC1 mutation identified by whole-exome sequencing. Gene 546: 425-429, 2014.

20. Mansouri M, Kayserili H, Elalaoui SC, Nishimura G, Iida A, Lyahyai J, Miyake N, Matsumoto N, Sefiani A and Ikegawa S: Novel DDR2 mutation identified by whole exome sequencing in a Moroccan patient with spondylo-meta-epiphyseal dysplasia, short limb-abnormal calcification type. Am J Med Genet A 170A: 460-465, 2016 